\title{
Produtividade e qualidade da framboeseira 'Batum' cultivada sob cobertura plástica e dois espaçamentos em região subtropical
}

\author{
Production and quality of raspberries 'Batum' cultivated \\ under cover plastic and two spacing in subtropical area
}

\begin{abstract}
Paula Nogueira Curi ${ }^{\mathrm{I}}$ Rafael Pio $^{\mathrm{I}^{*}}$ Pedro Henrique Abreu Moura ${ }^{\mathrm{I}}$ Pedro Henrique Assis Sousa Gabriella Donato Bonfim ${ }^{I}$ Pedro Augusto de Oliveira Silva ${ }^{I}$
\end{abstract}

\section{RESUMO}

Framboesa é uma pequena fruta de grande importância econômica, que contém numerosos compostos fenólicos, com potenciais benéficos à saúde, porém, quando cultivada em regiões subtropicais possui baixa produtividade. Objetivou-se, neste trabalho, avaliar a produtividade de framboeseiras 'Batum', produzidas com ou sem cobertura plástica sobre o dossel das plantas, em duas densidades de plantio, sendo 0,25m e 0,5m entre plantas e 3,0m entre linhas e avaliadas por dois ciclos de produção (2012/13 e 2013/14), em região subtropical. $O$ delineamento experimental adotado foi em blocos ao acaso, em esquema fatorial $2 \times 2 \times 2$, com 6 blocos e 10 plantas por parcela. Foram avaliadas as características produtivas como número de frutos por planta, produção por planta e produtividade estimada. Além disso, foram demarcados os índices fenológicos, análises vegetativas e fisiológicas. $O$ menor espaçamento de plantio entre as plantas $(0,25 \mathrm{~m})$ e o plantio sem cobertura plástica sobre o dossel das plantas resultaram em maior produtividade das framboseiras 'Batum'.

Palavras-chave: Rubus idaeus L., produção, plasticultura, densidade.

\section{ABSTRACT}

Raspberry is an economically important berry crop, its fruit contains numerous phenolic compounds with potential health benefits, however ver, in subtropical areas has been low production. The objective of this study was to evaluate the productivity of raspberry 'Batum' produced with or without plastic cover over the plant canopy and two planting densities, being $0.25 \mathrm{~m}$ and $0.5 \mathrm{~m}$ between plants and $3.0 \mathrm{~m}$ between rows and cycles (2012/13 and 2013/14), in subtropical area. The experimental design was randomized blocks, factorial $2 \times 2 \times 2$ with 6 blocks and 10 plants per plot. It was evaluated the productive plant characteristics such as number of fruits per plant, production per plant and productivity estimated. Moreover, phenological data, vegetative and physiologic analysis. The planting densities at $0.25 \mathrm{~m}$ between plants and without plastic cover over the plant canopy result in increased productivity of raspberry 'Batum'.

Key words: Rubus idaeus L., production, plasticulture, density.

\section{INTRODUÇ̃̃O}

O interesse pelo cultivo de framboesas tem crescido no Brasil nos últimos cinco anos, principalmente em regiões subtropicais, como no sul de Minas, na Serra da Mantiqueira e em alguns locais do estado do Paraná (CAMPAGNOLO \& PIO, 2012; SILVA et al., 2012; SOUZA et al., 2014a). Quando cultivadas em regiões subtropicais, as framboesas apresentam maior qualidade, devido ao aumento de $\beta$-caroteno e na atividade antioxidante (MARO et al., 2013; SOUZA et al., 2014b).

As framboeseiras cultivadas em regiões subtropicais permanecem em produção entre os meses de novembro a maio, sendo considerado um único período de colheita, diferentemente do que ocorre em regiões mais frias, onde se tem dois períodos de colheita bem definidos, um em pleno verão e outro no início do outono (MARO et al., 2012).

A cultivar que vem se destacando nas regiões subtropicais é a 'Batum', no entanto, a

\footnotetext{
'Departamento de Agricultura, Universidade Federal de Lavras (UFLA), CP 3037, 37200-000, Lavras, MG, Brasil. E-mail: rafaelpio@dag.ufla.br. *Autor para correspondência. 
produtividade das plantas ainda é baixa, atingindo, no máximo, 5t ha ${ }^{-1}$ (MOURA et al., 2012; MARO et al., 2014). Assim, uma alternativa seria o aumento da densidade de plantio, através da redução do espaçamento entre as plantas no plantio, o que poderia ocasionar aumento da produtividade dos pomares.

Uma alternativa visando à diminuição das chuvas sobre as folhas e frutos é a instalação de uma cobertura plástica sobre o dossel das plantas. Em vinhedos, essa medida já é utilizada visando conter os efeitos do excesso de precipitação, principalmente na época da maturação, para obtenção de uvas de maior qualidade e livre de patógenos (COMIRAN et al., 2012). Outras vantagens da utilização da cobertura plástica referem-se à proteção das plantas aos danos causados por granizo (HERNANDES et al., 2013). Assim, a cobertura plástica sobre o dossel das plantas poderia melhorar a qualidade das framboesas, tornando-as com maiores dimensões e com menores perdas, em razão da incidência de chuvas em demasia e granizo, que deterioram as framboesas durante o verão na região sudeste brasileira.

Nesse sentido, objetivou-se com o presente trabalho verificar a influência da cobertura plástica e do espaçamento na produtividade estimada da framboeseira 'Batum' em dois ciclos de produção, em região subtropical.

\section{MATERIAL E MÉTODOS}

O trabalho foi realizado no Setor de Fruticultura do Departamento de Agricultura (DAG) da Universidade Federal de Lavras (UFLA), LavrasMG, no período de março de 2012 a maio de 2014 . O referido município está situado a $21^{\circ} 14^{\prime}$ de latitude Sul e $45^{\circ} 00^{\prime}$ de longitude Oeste, a uma altitude média de 918 metros. O clima da região é do tipo Cwb (clima mesotérmico ou tropical de altitude), com inverno seco e verão chuvoso, segundo a classificação de Köppen (MOURA et al., 2012).

Mudas da cultivar de framboeseira 'Batum' foram transplantadas a campo em dois espaçamentos, $3 \mathrm{~m}$ entre linhas $\mathrm{x} 0,25 \mathrm{~m}$ entre plantas e $3 \mathrm{~m}$ entre linhas x $0,5 \mathrm{~m}$ entre plantas, em janeiro de 2012, em quatro linhas de plantio, sendo duas em cada espaçamento, contendo 60 plantas cada linha de plantio. Por ocasião do plantio, foram aplicados três litros de compostagem, $200 \mathrm{~g}$ de calcário e $100 \mathrm{~g}$ de superfosfato simples por metro linear na linha de plantio. As plantas foram conduzidas em palanques constituídos por dois mourões de eucalipto a uma altura de $1,5 \mathrm{~m}$ do solo e espaçados a $0,60 \mathrm{~m}$, com arames lisos paralelos, localizados a $0,60 \mathrm{~m}$ e $1,20 \mathrm{~m}$ do solo.

Das duas linhas de plantio em cada espaçamento, uma linha permaneceu sem a cobertura plástica sobre o dossel. Já na outra linha de plantio, as plantas foram cobertas sobre o dossel, por plástico leitoso de $150 \mathrm{~m} \mu$ de espessura. A cobertura plástica foi instalada sobre fios metálicos e arcos de ferro galvanizado com 1,43m de largura, dispostos no dossel das framboeseiras a cada $2,5 \mathrm{~m}$, os quais, por sua vez, foram fixados sobre a estrutura de postes de eucalipto, que propiciou a sustentação e forma ao sistema de condução. O ponto mais alto da cobertura plástica ficou a uma distância de $0,70 \mathrm{~m}$, em relação às extremidades das hastes.

Durante a condução das plantas, os tratos culturais foram realizados segundo as recomendações de MOURA et al. (2012), sendo as podas drásticas de redução realizadas durante o período de dormência das plantas (primeira quinzena de junho). Todas as plantas foram irrigadas por gotejadores espaçados a cada $0,30 \mathrm{~m}$, com vazão de 1,7 litros por hora a cada gotejador, por um período de quatro horas, durante o dia, ao longo de todo o período experimental.

$\mathrm{O}$ delineamento adotado foi em blocos ao acaso, em esquema fatorial $2 \times 2 \times 2$. O primeiro fator foi composto pelos diferentes espaçamentos entre plantas $(0,25 \mathrm{~m}$ e $0,5 \mathrm{~m})$, o segundo fator pelo uso ou não da cobertura sobre o dossel das plantas e o terceiro fator pelos dois ciclos de produção (2012/13 e 2013/14), utilizando-se seis blocos e 10 plantas por parcela.

Nos dois ciclos de produção, foi avaliado o comportamento fenológico das plantas, indicando: o início da brotação, quando se iniciou a emissão das brotações no solo, oriundo das raízes; início, término e duração da florada, através das anotações, em datas, do início da emissão das flores, final da emissão das flores e a duração, sendo essa calculada através do somatório dos dias em que as plantas permaneceram em floração; e, início, término e duração da colheita, através das anotações, em datas, do início da colheita de frutos maduros, final da colheita e a duração, sendo essa calculada através do somatório dos dias em que as plantas permaneceram com frutos maduros, que foram colhidos. No caso da duração da florada e colheita, foi realizada análise estatística. Foram avaliadas as variáveis produtivas, como número de frutos por planta, produção por planta $\left(\mathrm{g}\right.$ planta $\left.^{-1}\right)$ 
e produtividade estimada ( $\left.\mathrm{t} \mathrm{ha}^{-1}\right)$. Os frutos foram colhidos três vezes por semana, contados e a massa obtida com o auxílio de uma balança semianalítica digital. Ao final do ciclo de produção, somaram-se todos os frutos e todas as massas registradas para, assim, determinar a produção por planta e estimar a produtividade. Durante a fase experimental, foram coletadas as temperaturas máximas, mínimas e precipitação para os meses março de 2012 a maio de 2014 (Figura 1).

No primeiro ciclo de produção, avaliouse a qualidade dos frutos e o crescimento das plantas. Foram marcados dez ramos por parcela, na qual foram medidos o comprimento e diâmetro das hastes, além da contagem do número de rebentos, por planta e por metro linear. Para a realização das análises qualitativas massa média dos frutos, com auxílio de uma balança semianalítica, modelo
AUX220 (Shimadzu), número médio de drupéolos, comprimento e diâmetro médio dos frutos, com o auxílio de um paquímetro digital 150mm (Kingtools), foram utilizados 20 frutos de cada parcela.

Em pleno período de produção, foi avaliado o comportamento fisiológico das plantas através da determinação da fotossíntese líquida e condutância estomática, utilizando um analisador de gás a infravermelho (IRGA, model LI-6400, LI-Cor, USA). A avaliação foi realizada em dia límpido, em dezembro de 2013, entre $8 \mathrm{~h}$ e $11 \mathrm{~h} 30 \mathrm{~min}$, em dez folhas por parcela, utilizando sempre uma folha madura do terço médio não sombreada por outras folhas. As mesmas folhas selecionadas para análise do IRGA foram utilizadas na determinação da medida indireta da clorofila, realizada utilizando o medidor portátil de clorofila, modelo SPAD-502. As leituras foram

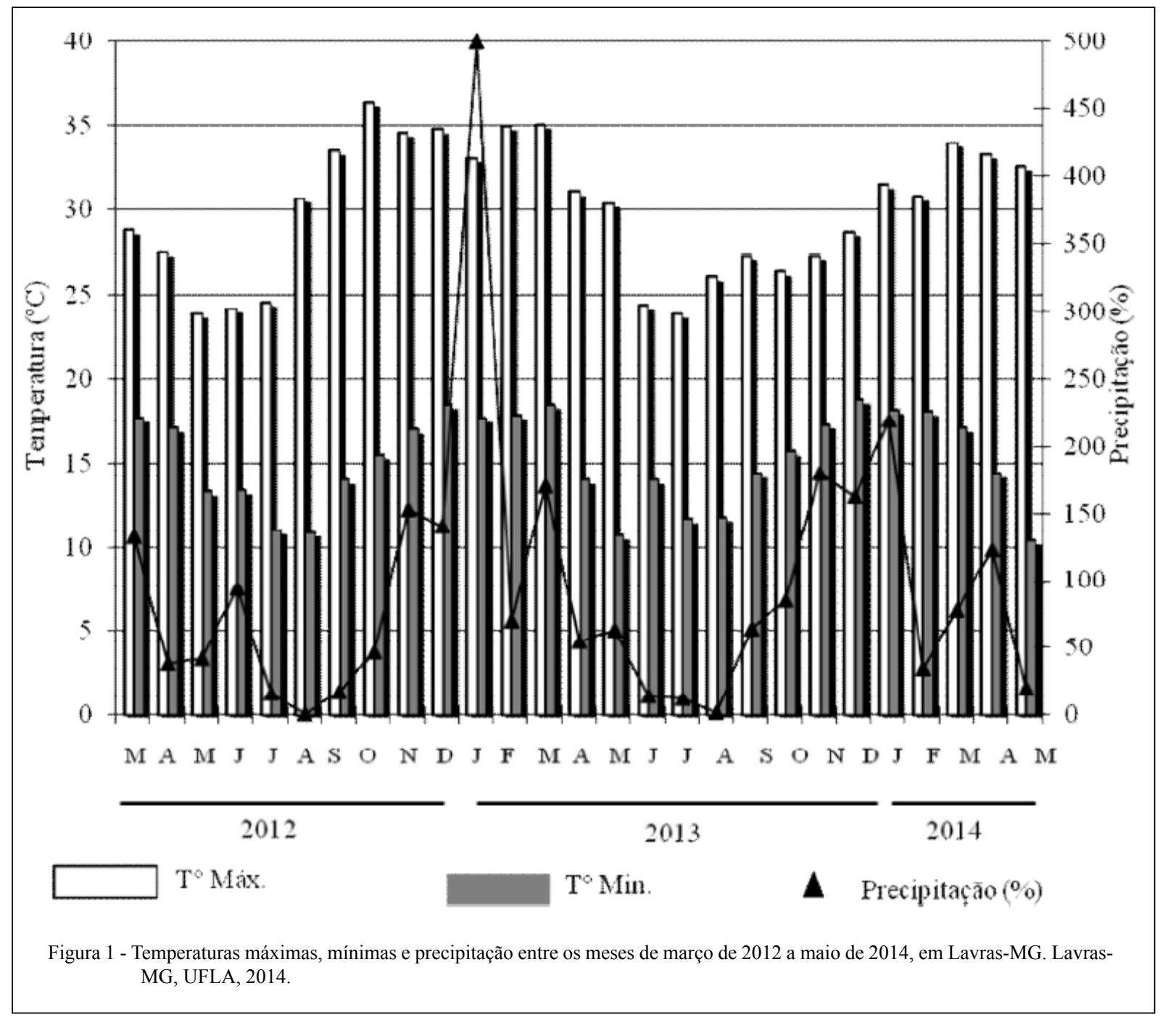

Ciência Rural, v.45, n.11, nov, 2015. 
feitas, aproximadamente, no centro das folhas, em apenas um lado da nervura (distância fixada pelo regulador de profundidade do aparelho). As coletas de campo para o índice de área foliar foram realizadas com o aparelho modelo LAI2200. Para tal, foi utilizado um sensor ótico (LAI2250), acoplado ao equipamento com um view cap de $90^{\circ}$.

Ao final de todas as análises, as quantificações realizadas foram submetidas à análise de variância e as médias comparadas pelo teste de Tukey, ao nível de 5\% de probabilidade.

\section{RESULTADOS E DISCUSSÃO}

Pelas avaliações fenológicas, não houve diferença entre as fenofases nas linhas com espaçamentos distintos, ao contrário das plantas alocadas com e sem cobertura sobre o dossel. Nos dois ciclos de produção, as plantas iniciaram a brotação, com emissão dos rebentos oriundos do sistema radicular em meados de julho (Tabela 1). $\mathrm{O}$ florescimento foi mais intenso entre setembro e maio, com as plantas permanecendo em floração ao redor de 240 dias, independente das plantas estarem cobertas ou não. Diferentemente do que ocorreu no oeste Paranaense, de acordo com MOURA et al. (2012), onde o florescimento da framboeseira 'Batum' se iniciou entre final de julho e início de agosto.

Como os frutos da framboeseira 'Batum' possuem frutificação em torno de 20 dias, ou seja, da abertura floral à colheita do fruto (MARO et al., 2012), as colheitas se concentraram entre os meses de outubro a maio (Tabela 1). Apenas no primeiro ciclo de produção, as plantas sem cobertura sobre o dossel apresentaram maior período de colheita (245 dias).
Quanto ao desempenho produtivo das plantas, não houve interação entre os tratamentos, apenas diferença estatística para os fatores isolados. No primeiro ciclo de produção $(2012 / 13)$, as framboeseiras produziram mais frutos e, consequentemente, registraram maior produção por planta e produtividade estimada. A produtividade estimada no primeiro ciclo de produção foi de $10,3 \mathrm{t}$ $\mathrm{ha}^{-1}$, sendo 2,9t ha-1 a mais em relação ao segundo ciclo de produção (Tabela 2). Essa diferença é explicada pela grande variação da precipitação registrada entre os períodos, haja vista que a precipitação entre os meses de janeiro e março em 2013 foi superior ao mesmo período do ano de 2014 (Figura 1), o que comprometeu a emissão das hastes secundárias e a brotação das gemas subapicais, via redução da umidade relativa do ar. Segundo MARO et al. (2012), a emissão das hastes secundárias e a brotação das gemas subapicais são responsáveis pela produção de frutos do outono das framboeseiras, ou seja, as colheitas concentradas entre o final de março e início de junho.

Plantas sem a cobertura sobre o dossel apresentaram maior produção por planta e produtividade estimada de framboesas (Tabela 2). Apesar de não ter ocorrido diferença estatística nas avaliações de crescimento das framboeseiras (número de rebentos por planta e por metro linear, comprimento e diâmetro das hastes), entre plantas descobertas e com cobertura sobre o dossel, a razão pelo fato de plantas descobertas terem registrado maior produção de frutos pode ser explicada pelas variáveis fisiológicas avaliadas. Plantas dispostas sem cobertura sobre o dossel apresentaram maior área foliar, taxa fotossintética líquida e condutância estomática, o que promoveu melhoria na eficiência fotossintética das plantas (Tabela 3).

Tabela 1 - Descrição fenológica nos ciclo de produção 2012/13 e 2013/14 - início da brotação (IB), início, término e duração da florada (IF, TF e DF) e colheita (IC, TC e DC) da framboeseira 'Batum', cultivada com e sem cobertura plástica sobre o dossel das plantas. Lavras-MG, UFLA, 2014.

\begin{tabular}{|c|c|c|c|c|c|c|c|}
\hline Cobertura plástica & IB & IF & $\mathrm{TF}$ & $\mathrm{DF}^{*}$ & IC & $\mathrm{TC}$ & $\mathrm{DC}^{*}$ \\
\hline Sem & $05 / 07$ & $\begin{array}{l}----C \\
10 / 09\end{array}$ & $\begin{array}{c}\text { roduçã } \\
13 / 05\end{array}$ & $\begin{array}{l}3 \text { - } \\
246 \mathrm{a}\end{array}$ & $01 / 10$ & 03/06 & $245 a$ \\
\hline Com & $05 / 07$ & $17 / 09$ & $17 / 05$ & $243 \mathrm{a}$ & $15 / 10$ & $07 / 06$ & $235 \mathrm{~b}$ \\
\hline $\mathrm{CV}(\%)$ & - & - & - & 2,76 & - & - & 1,98 \\
\hline Sem & $01 / 07$ & $19 / 09$ & $06 / 05$ & $230 \mathrm{a}$ & $05 / 10$ & $20 / 05$ & $227 \mathrm{a}$ \\
\hline Com & $26 / 06$ & $20 / 09$ & $16 / 05$ & $239 a$ & $16 / 10$ & $30 / 05$ & $226 \mathrm{a}$ \\
\hline $\mathrm{CV}(\%)$ & - & - & - & 5,21 & - & - & 2,77 \\
\hline
\end{tabular}

${ }^{*}$ Médias seguidas da mesma letra não diferem entre si pelo teste de Tukey a 5\% de probabilidade. 
Tabela 2 - Número de frutos por planta, produção e produtividade estimada em framboeseiras 'Batum' cultivadas com e sem cobertura plástica sobre o dossel das plantas e em dois espaçamentos de plantio, nos ciclos de produção 2012/13 e 2013/14. Lavras-MG, UFLA, 2014.

\begin{tabular}{|c|c|c|c|c|c|c|c|}
\hline \multirow[t]{2}{*}{ Avaliações } & \multicolumn{2}{|c|}{ Ciclos de produção* } & \multicolumn{2}{|c|}{$\begin{array}{c}\text { Cobertura plástica sobre } \\
\text { o dossel das plantas* }\end{array}$} & \multicolumn{2}{|c|}{$\begin{array}{c}\text { Espaçamentos de plantio } \\
\text { entre plantas* }\end{array}$} & \multirow[t]{2}{*}{$\mathrm{CV}(\%)$} \\
\hline & $2012 / 13$ & $2013 / 14$ & Com & Sem & $3 \times 0,25 \mathrm{~m}$ & $3 \times 0,50 \mathrm{~m}$ & \\
\hline Número de frutos por planta & $491 \mathrm{a}$ & $302 b$ & $352 b$ & $444 a$ & $324 b$ & $480 \mathrm{a}$ & 17,05 \\
\hline Produção (g planta $\left.{ }^{-1}\right)$ & $1.116,8 \mathrm{a}$ & $742,3 b$ & $870,5 b$ & $996,6 \mathrm{a}$ & $773,4 b$ & $1.108,6 \mathrm{a}$ & 19,77 \\
\hline Produtividade estimada $\left(\mathrm{t} \mathrm{ha}^{-1}\right)$ & $10,3 \mathrm{a}$ & $7,4 \mathrm{~b}$ & $8,2 b$ & $9,5 \mathrm{a}$ & $10,3 \mathrm{a}$ & $7,4 \mathrm{~b}$ & 19,76 \\
\hline
\end{tabular}

"Médias seguidas da mesma letra na linha não diferem entre si pelo teste de Tukey, a 5\% de probabilidade.

Como as framboesas são produzidas em panículas (MARO et al., 2012), a eficiência das folhas para a relação fonte/dreno preconizou o ganho de massa e o aumento dos frutos produzidos nas plantas descobertas, em relação às plantas que continham cobertura sobre o dossel.

De acordo com as análises de qualidade dos frutos, não houve diferença entre o diâmetro dos frutos, porém, o número de drupéolos, massa unitária dos frutos e comprimento foram maiores em plantas dispostas sobre a cobertura plástica (Tabela 3), indicando aumento da qualidade das framboesas com o uso da cobertura plástica, concordando com COMIRAN et al. (2012).

Esses resultados concordam com HERNANDES et al. (2013), que verificaram que as bagas das uvas colhidas em plantas sob cobertura plástica possuem maior quantidade de sólidos solúveis e qualidade em relação às frutas colhidas em plantas sem o uso da cobertura sobre o dossel. Essa diferença pode ser devido à menor incidência de gotas de chuvas e também pela diminuição da incidência direta dos raios solares sobre as frutas.

Quanto aos espaçamentos adotados, plantas menos adensadas $(3 \times 0,50 \mathrm{~m})$ produziram mais frutos, $\mathrm{o}$ que proporcionou um aumento na produção por planta de $335,2 \mathrm{~g} \mathrm{planta}^{-1}$, em relação às plantas dispostas no espaçamento $3 \times 0,25 \mathrm{~m}$ (Tabela 2). Segundo as avaliações de qualidade de fruto, framboeseiras dispostas no espaçamento $3 \times 0,50 \mathrm{~m}$ produziram frutos com maior número de drupéolos e, consequentemente, maior massa unitária (Tabela 3). Isso porque, nesse espaçamento mais amplo, o número de rebentos por planta foi superior em relação às plantas dispostas

Tabela 3 - Número de drupéolos, massa, comprimento e diâmetro do fruto, número de rebentos por planta e por metro, comprimento $€$ diâmetro das hastes, área foliar, taxa fotossintética líquida, condutância estomática e teor de clorofila em framboeseiras 'Batum', cultivadas com e sem cobertura plástica sobre o dossel das plantas e em dois espaçamentos de plantio. Lavras-MG, UFLA, 2014.

\begin{tabular}{|c|c|c|c|c|c|}
\hline \multirow{2}{*}{ Avaliações } & \multicolumn{2}{|c|}{$\begin{array}{l}\text { Cobertura plástica sobre o } \\
\text { dossel das plantas }\end{array}$} & \multicolumn{2}{|c|}{$\begin{array}{l}\text { Espaçamentos de plantio } \\
\text { entre plantas }\end{array}$} & \multirow{2}{*}{$\mathrm{CV}(\%)$} \\
\hline & Com* & Sem & $3 \times 0,25 \mathrm{~m}^{*}$ & $3 \times 0,5 \mathrm{~m}$ & \\
\hline Número de drupéolos & $65,66 \mathrm{a}$ & $59,16 \mathrm{~b}$ & $59,33 b$ & $65,5 \mathrm{a}$ & 2,92 \\
\hline Massa do fruto (g) & $3,00 \mathrm{a}$ & $2,86 \mathrm{~b}$ & $2,87 b$ & $3,00 \mathrm{a}$ & 5,06 \\
\hline Comprimento do fruto (mm) & $17,30 \mathrm{a}$ & $16,04 \mathrm{~b}$ & $17,23 \mathrm{a}$ & $16,12 b$ & 1,91 \\
\hline Diâmetro do fruto (mm) & $17,61 \mathrm{a}$ & $17,84 \mathrm{a}$ & $18,03 \mathrm{a}$ & $17,00 \mathrm{~b}$ & 10,04 \\
\hline Número de rebentos por planta & $11,57 \mathrm{a}$ & $12,66 \mathrm{a}$ & $10,33 b$ & $14,10 \mathrm{a}$ & 16,40 \\
\hline Número de rebentos por metro & $36,81 \mathrm{a}$ & $41,05 \mathrm{a}$ & $45,91 \mathrm{a}$ & $31,12 b$ & 12,92 \\
\hline Comprimento das hastes $(\mathrm{cm})$ & $77,63 a$ & $87,17 \mathrm{a}$ & $92,83 \mathrm{a}$ & $71,45 b$ & 22,19 \\
\hline Diâmetro das hastes (mm) & $6,66 a$ & $6,34 \mathrm{a}$ & $6,66 \mathrm{a}$ & $6,36 \mathrm{~b}$ & 6,08 \\
\hline Área foliar $\left(\mathrm{m}^{2} \mathrm{~m}^{-2}\right)$ & $3,20 b$ & $3,90 \mathrm{a}$ & $3,40 \mathrm{a}$ & $3,80 \mathrm{a}$ & 18,91 \\
\hline Taxa fotossintética líquida $\left(\mu \mathrm{mol} \mathrm{CO} \mathrm{Cm}^{-2} \mathrm{~s}^{-1}\right)$ & $16,70 \mathrm{~b}$ & $18,50 \mathrm{a}$ & $18,40 \mathrm{a}$ & $17,70 \mathrm{a}$ & 4,18 \\
\hline Condutância estomática $\left(\mathrm{mol} \mathrm{m}^{-2} \mathrm{~s}^{-1}\right)$ & $0,30 \mathrm{~b}$ & $0,40 \mathrm{a}$ & $0,30 \mathrm{a}$ & $0,30 \mathrm{a}$ & 5,02 \\
\hline Teor de clorofila & $36,60 \mathrm{a}$ & $38,50 \mathrm{a}$ & $37,00 \mathrm{a}$ & $38,10 \mathrm{a}$ & 10,89 \\
\hline
\end{tabular}

*Médias seguidas da mesma letra na linha não diferem entre si pelo teste de Tukey, a 5\% de probabilidade. 
no espaçamento menor. Ressalta-se que não houve diferença estatística nas avaliações fisiológicas entre as framboeseiras dispostas em ambos os espaçamentos (Tabela 3).

Por outro lado, devido às plantas dispostas no espaçamento de $3 \times 0,25 \mathrm{~m}$ propiciarem a alocação de 13.334 plantas ha $^{-1}$ e plantas no espaçamento $3 \times 0,50 \mathrm{~m}$ alocarem 6.667 plantas $\mathrm{ha}^{-1}$, houve diferença na produtividade estimada, chegando a atingir $10,3 \mathrm{t} \mathrm{ha}^{-1}$ em plantas no espaçamento menor entre plantas (Tabela 2). O número de rebentos por metro linear no espaçamento mais adensado $(3 \times 0,25 \mathrm{~m})$ foi superior em relação ao espaçamento mais amplo (14,8 rebentos a mais); o comprimento e o diâmetro das hastes também foram superiores em plantas dispostas no espaçamento $3 \times 0,25 \mathrm{~m}$ (Tabela 3 ). Por essas razões, houve aumento da produtividade estimada quando se adotou o espaçamento mais adensado no plantio das framboeseiras.

Esses resultados foram maiores do que a produtividade estimada de $5 \mathrm{t} \mathrm{ha}^{-1}$ da framboeseira 'Batum' relatado por MOURA et al. (2012). Acredita-se que, além do aumento da densidade das plantas, o uso da irrigação localizada possa ter contribuído para essa elevação do potencial produtivo da framboeseira 'Batum'. Segundo PAGOT (2006), a produtividade estimada dos plantios de framboeseira no Brasil é extremamente variável, sendo a maior obtida na região de Vacaria $\left(5,6 \mathrm{tha}^{-1}\right)$. No entanto, segundo PARRA-QUEZADA et al. (2007), a produtividade estimada de um pomar adequadamente manejado pode superar $10 \mathrm{t} \mathrm{ha}^{-1}$.

\section{CONCLUSÃO}

O menor espaçamento de plantio entre as plantas $(0,25 \mathrm{~m})$ e também o plantio sem cobertura plástica sobre o dossel das plantas resultam em maior produtividade das framboseiras 'Batum'.

\section{AGRADECIMENTOS}

Ao Conselho Nacional de Desenvolvimento Científico e Tecnológico (CNPq), Coordenação de Aperfeiçoamento de Pessoal de Nível Superior (CAPES) e a Fundação de Amparo à Pesquisa do Estado de Minas Gerais (FAPEMIG), pelo auxílio financeiro.

\section{REFERÊNCIAS}

CAMPAGNOLO, M.A.; PIO, R. Phenological and yield performance of black and redberry cultivars in western Paraná
State. Acta Scientiarum. Agronomy, Maringá, v.34, n.4, p.439-444, 2012. Disponível em: <http://www.scielo.br/scielo. php?pid $=$ S1807-86212012000400011\&script $=$ sci_arttext $>$. Acesso em: 20 jun. 2014. doi:10.4025/actasciagron.v34i4.15528.

COMIRAN, F. et al. Microclima e produção de videiras 'Niagara Rosada' em cultivo orgânico sob cobertura plástica. Revista Brasileira de Fruticultura, Jaboticabal, v.34, n.1, p.152-159, 2012. Disponível em: <http://www.scielo.br/ scielo.php?pid=S0100-29452012000100021\&script $=$ sci arttext>. Acesso em: 30 jun. 2014. doi:10.1590/S010029452012000100021.

HERNANDES, J.L et al. Comportamento produtivo da videira 'Niagara Rosada' em diferentes sistemas de condução, com e sem cobertura plástica, durante as safras de inverno e de verão. Revista Brasileira de Fruticultura, Jaboticabal, v.35, n.1, p.123-130, 2013. Disponível em: <http://www.scielo.br/ scielo.php?script $=$ sci_arttext\&pid $=$ S0 10029452013000100 018\&lng=pt\&nrm=iso\&tlng=en $>$. Acesso em: 25 jun. 2014. doi:10.1590/S0100-29452013000100018.

MARO, L.A.C. et al. Environmental and genetic variation in the post-harvest quality of raspberries in subtropical areas in Brazil. Acta Scientiarum. Agronomy, Maringá, v.36, n.3, p.323-328, 2014. Disponível em: <http://periodicos.uem.br/ ojs/index.php/ActaSciAgron/article/view/18050>. Acesso em: 01 jul. 2014. doi:http://dx.doi.org/10.4025/actasciagron. v36i3.18050.

MARO, L.A.C. et al. Bioactive compounds, antioxidant activity and mineral composition of fruits of raspberry cultivars grown in subtropical areas in Brazil. Fruits, Montpellier, v.68, n.3, p.209-217, 2013. Disponível em: <http://journals.cambridge. org/action/displayAbstract? fromPage $=$ online \&aid $=8934317 \&$ fulltext Type $=$ RA\&fileId $=S 0248129413000686>$. Acesso em: 28 jun. 2014. doi: $10.1051 /$ fruits/2013068.

MARO, L.A.C. et al. Ciclo de produção de cultivares de framboeseiras (Rubus idaeus) submetidas à poda drástica nas condições do sul de Minas Gerais. Revista Brasileira de Fruticultura, Jaboticabal, v.34, n.2, p.435-441, 2012. Disponível em: $<$ http://www.scielo.br/scielo.php?script=sci arttext\&pid $=\mathrm{S} 0100-29452012000200016>$. Acesso em: 28 maio 2014. doi:10.1590/S0100-29452012000200016.

MOURA, P.H.A. et al. Fenologia e produção de cultivares de framboeseiras em regiões subtropicais no Brasil. Pesquisa Agropecuária Brasileira, Brasília, v.47, n.12, p.1714-1721, 2012. Disponível em: <http://www.scielo.br/ scielo.php?pid=S0100-204X2012001200006\&script $=$ sci arttext>. Acesso em: 10 jul. 2014. doi:10.1590/S0100204X2012001200006.

PAGOT, E. Cultivo de pequenas frutas: amora-preta, framboesa e mirtilo. Porto Alegre: EMATER/RS-ASCAR, 2006. 41p. Disponível em: <http:/www.bdpa.cnptia.embrapa.br/busca?b=ad $\&$ id $=743625 \&$ biblioteca $=$ vazio \&busca $=$ autoria:\%22PAGOT, $\% 20$ E.\%22\&qFacets=autoria:\%22PAGOT,\%20E.\%22\&sort=\&pagina $\mathrm{cao}=\mathrm{t} \&$ paginaAtual $=1>$. Acesso em: 11 jul. 2014.

PARRA-QUEZADA, R.Á. et al. Efecto de fecha y tipo de poda em frambuesa roja "Malling autumn Bliss". Revista Chapingo Serie Horticultura, Chapingo, v.13, n.2, p.201-206, 2007. Disponível em: $<$ http://www.redalyc.org/pdf/609/60913280013. pdf $>$. Acesso em: 05 jul. 2014 
SILVA, K.N. et al. Produção de mudas de framboeseira negra por diferentes métodos de propagação vegetativa. Ciência Rural, Santa Maria, v.42, n.3, p.418-422, 2012. Disponível em: $<$ http://www.scielo.br/scielo.php?script=sci_arttext\& pid=S0103-84782012000300006>. Acesso em: 01 jul. 2014. doi:10.1590/S0103-84782012000300006.

SOUZA, V.R. et al. Determination of the bioactive compounds, antioxidant activity and chemical composition of Brazilian blackberry, red raspberry, strawberry, blueberry and sweet cherry fruits. Food Chemistry, Reading, v.156, p.362-368, 2014a. Disponível em: <http://ac.els-cdn.com/S0308814614001770/1-
s2.0-S0308814614001770-main.pdf?_tid=f1425554-bbf4-11e3aa89-00000aab0f6b\&acdnat $=1396614824 \_0$ da62 cdedaf5c9f8 5864d781725edb24>. Acesso em: 01 jul. 2014. doi:10.1016/j. foodchem.2014.01.125.

SOUZA, V.R. et al. Evaluation of the jelly processing potential of raspberries adapted in Brazil. Journal of Food Science, Chicago, v.79, n.3, p.S407-S412, 2014b. Disponível em: $\quad<$ http://onlinelibrary.wiley.com/doi/10.1111/17503841.12354/abstract; jsessionid=1B0D300619EBE3AFF 13803C2932FC889.f04t03>. Acesso em: 11 maio. 2015. doi:10.1111/1750-3841.12354. 